\title{
A microfluidic platform for the rapid determination of distribution coefficients by gravity assisted droplet-based liquid-liquid extraction
}

Poulsen, Carl Esben; Wootton, Robert C. R. ; Wolff, Anders; deMello, Andrew J. ; Elvira, Katherine S.

Published in:

Analytical Chemistry

Link to article, DOI:

10.1021/acs.analchem.5b01061

Publication date:

2015

Link back to DTU Orbit

Citation (APA):

Poulsen, C. E., Wootton, R. C. R., Wolff, A., deMello, A. J., \& Elvira, K. S. (2015). A microfluidic platform for the rapid determination of distribution coefficients by gravity assisted droplet-based liquid-liquid extraction. Analytical Chemistry, 87(12), 6265-6270. https://doi.org/10.1021/acs.analchem.5b01061

\section{General rights}

Copyright and moral rights for the publications made accessible in the public portal are retained by the authors and/or other copyright owners and it is a condition of accessing publications that users recognise and abide by the legal requirements associated with these rights.

- Users may download and print one copy of any publication from the public portal for the purpose of private study or research.

- You may not further distribute the material or use it for any profit-making activity or commercial gain

- You may freely distribute the URL identifying the publication in the public portal 
Article

Subscriber access provided by DTU Library

\section{A microfluidic platform for the rapid determination of distribution coefficients by gravity assisted droplet-based liquid-liquid extraction}

Carl Esben Poulsen, Robert C.R. Wootton, Anders Wolff, Andrew J. deMello, and Katherine S. Elvira

Anal. Chem., Just Accepted Manuscript • DOI: 10.1021/acs.analchem.5b01061 • Publication Date (Web): 18 May 2015

Downloaded from http://pubs.acs.org on May 22, 2015

\section{Just Accepted}

"Just Accepted" manuscripts have been peer-reviewed and accepted for publication. They are posted online prior to technical editing, formatting for publication and author proofing. The American Chemical Society provides "Just Accepted" as a free service to the research community to expedite the dissemination of scientific material as soon as possible after acceptance. "Just Accepted" manuscripts appear in full in PDF format accompanied by an HTML abstract. "Just Accepted" manuscripts have been fully peer reviewed, but should not be considered the official version of record. They are accessible to all readers and citable by the Digital Object Identifier (DOI®). "Just Accepted" is an optional service offered to authors. Therefore, the "Just Accepted" Web site may not include all articles that will be published in the journal. After a manuscript is technically edited and formatted, it will be removed from the "Just Accepted" Web site and published as an ASAP article. Note that technical editing may introduce minor changes to the manuscript text and/or graphics which could affect content, and all legal disclaimers and ethical guidelines that apply to the journal pertain. ACS cannot be held responsible for errors or consequences arising from the use of information contained in these "Just Accepted" manuscripts. 


\title{
A microfluidic platform for the rapid
}

\section{determination of distribution coefficients by} gravity assisted droplet-based liquid-liquid

\section{extraction $^{\dagger}$}

\author{
Carl Esben Poulsen, ${ }^{\ddagger}$ Robert C. R. Wootton, " Anders Wolff, $\ddagger$ Andrew J. \\ deMello, and Katherine S. Elvira*, \\ Department of Micro- and Nanotechnology, Technical University of Denmark, Kgs. Lyngby, \\ Denmark, and Institute of Chemical and Bioengineering, Department of Chemistry and \\ Applied Biosciences, ETH Zurich, Switzerland. \\ E-mail: katherine.elvira@chem.ethz.ch
}

\begin{abstract}
The determination of pharmacokinetic properties of drugs, such as the distribution coefficient, $D$, is a crucial measurement in pharmaceutical research. Surprisingly, the conventional (gold standard) technique used for $D$ measurements, the shake-flask method, is antiquated and unsuitable for the testing of valuable and scarce drug candidates. Herein we present a simple microfluidic platform for the determination of distribution coefficients using droplet-based liquid-liquid extraction. For simplicity, this platform makes use of gravity to enable phase separation for analysis and is 48 times

${ }^{\dagger}$ Additional information is available in the electronic Supporting Information.

${ }^{*}$ To whom correspondence should be addressed

${ }^{\ddagger}$ DTU Nanotech

『ETH Zurich
\end{abstract}


faster and uses $99 \%$ less reagents than performing an equivalent measurement using the shake-flask method. Furthermore, the $D$ measurements achieved in our platform are in good agreement with literature values measured using traditional shake-flask techniques. Since $D$ is affected by volume ratios, we use the apparent acid dissociation constant, $p K^{\prime}$, as a proxy for inter-system comparison. Our platform determines a $p K$ ' value of $7.24 \pm 0.15$, compared to $7.25 \pm 0.58$ for the shake-flask method in our hands and 7.21 for the shake-flask method in literature. Devices are fabricated using injection moulding, the batch-wise fabrication time is less than 2 minutes per device (at a cost of 1 USD per device) and the inter-device reproducibility is high.

\section{Introduction}

The pharmacokinetic properties of drugs are key indicators of how a drug will perform in the human body. The aim behind the study of these properties is to allow prediction of the viability of a drug prior to its administration to human subjects so that the cost and high attrition rates associated with drug discovery can be mitigated. One of the most commonly used metrics to determine pharmacokinetic properties is the distribution coefficient, $D$, which is an important indicator of the interaction between a compound and cellular membranes, and hence allows the prediction of the extent of absorption of orally administered drugs.

The standard experimental methodology for measuring distribution coefficients is the shake-flask method, where the distribution of a drug between a hydrophilic (aqueous) phase and a hydrophobic phase (normally octanol) is measured through use of a separating funnel or centrifuge. ${ }^{1}$ The advantages associated with this method are historical and based on the experimental simplicity and availability of the required equipment in normal laboratory settings. However, such measurements take a long time since the phases must be preequilibrated with the opposite phase over the course of 24 hours, ${ }^{1}$ and large volumes are required, which is problematic when assaying valuable drug samples. ${ }^{2}$ Furthermore, $D$ is known to be sensitive to contaminants. ${ }^{1}$ 
The adoption of microfluidic technologies provides interesting alternatives to the shakeflask method. Microfluidic platforms specialise in the manipulation of fluids on the microscale, with key advantages related to reduced sample sizes, enhanced assay speed and increased control over physical and chemical characteristics associated with the system under study. ${ }^{3}$ However, microfluidic platforms have not become de rigueur for distribution coefficient measurements due to factors related to the material used for fabrication, phase control and overall system complexity. For example, microfluidic platforms presented in the literature have been fabricated in materials that scale poorly or are too expensive for commercial scale production, such as PDMS, ${ }^{4-7}$ glass,${ }^{8-10}$ silicon, ${ }^{11,12}$ and polymers (such as thiolene, ${ }^{13}$ SIFEL $^{13}$ or NOA81 ${ }^{14}$ ) or require surface modification ${ }^{5}$ prior to use. Both these characteristics make commercial application of such platforms unfeasible. Furthermore, many microfluidic platforms have used the co-flow of oil and water phases through a device, ${ }^{4,11,13}$ which requires complicated structures for phase separation and stabilisation (such as the use of porous fluoropolymer membranes ${ }^{2,11}$ ), or relies on surface modification of wetting characteristics to stabilise the co-flowing phases. ${ }^{5,8,13}$

An alternative microfluidic method for liquid-liquid extraction makes use of droplets, which maximise the available surface area for the partitioning of compounds between the immiscible phases. These systems have the advantage of allowing easy removal of artefacts since each droplet defines a single measurement and hence contaminants are easily identified. Droplet-based microfluidic systems are in fact closely related to the shake-flask method, where phase agitation causes emulsification prior to phase separation by allowing the mixture to re-equilibrate using gravity. Microfluidic droplet-based systems however tend to use surfactants to stabilise $\operatorname{droplets}^{15}$ and hence require complicated techniques to drive de-emulsification for phase separation, such as the use of strong electric fields, ${ }^{12}$ or require complicated detection methods to perform in-droplet measurements, such as laser-induced fluorescence. ${ }^{9}$

It is clear that microfluidic platforms have the potential to revolutionise the measurement 
of distribution coefficients, especially for high-value drugs, and to ensure that these measurements are user-independent and considerably faster than conventional methods. However, for microfluidic platforms to achieve this goal they must be easy to use, cheap to produce commercially, of simple design, disposable, and provide a clear advantage over the shake-flask technique. The use of microfluidic technologies in commercial applications is an enduring goal in the field, but very few microfluidic platforms are commercially viable, specifically due to factors such as the material used for fabrication and ease of use. Herein we present a droplet-based microfluidic platform for the determination of distribution coefficients using gravity to separate the phases for analysis. The platform is fabricated using injection moulding and can be easily integrated into a production line. The devices are low-cost and hence disposable, which we demonstrate through the analysis of data gathered using over 30 devices. The material used for device fabrication is compatible with the solvent systems used for distribution coefficient measurements and we show that our microfluidic platform performs in a superior manner to the shake-flask method, specifically where cost, reagent volume and experimental time are paramount.

\section{Materials and Methods}

\subsection{Design of the microfluidic platform}

The microfluidic platform was designed to maximise the interaction between the aqueous and oil phases and ensures optimal mass transfer through the use of aqueous-in-oil droplets. After creation at a flow focusing geometry, droplets enter the separation chamber (Figure 1, Video S1). Extended droplet residence times in this chamber allow the droplets to travel a significant portion of the chamber width $(w)$ relative to the continuous phase due to gravity (further details of these calculations can be found in Section S1 of the Supporting Information, SI), hence allowing the removal of aqueous droplets from the continuous oil phase for further analysis of the oil phase. In other words, gravitational forces allow aqueous 
droplets to settle at the bottom of the separation chamber and hence exit at the lower, aqueous, outlet. This allows the droplet-free oil phase to be collected from the upper outlet and used for absorption measurements.

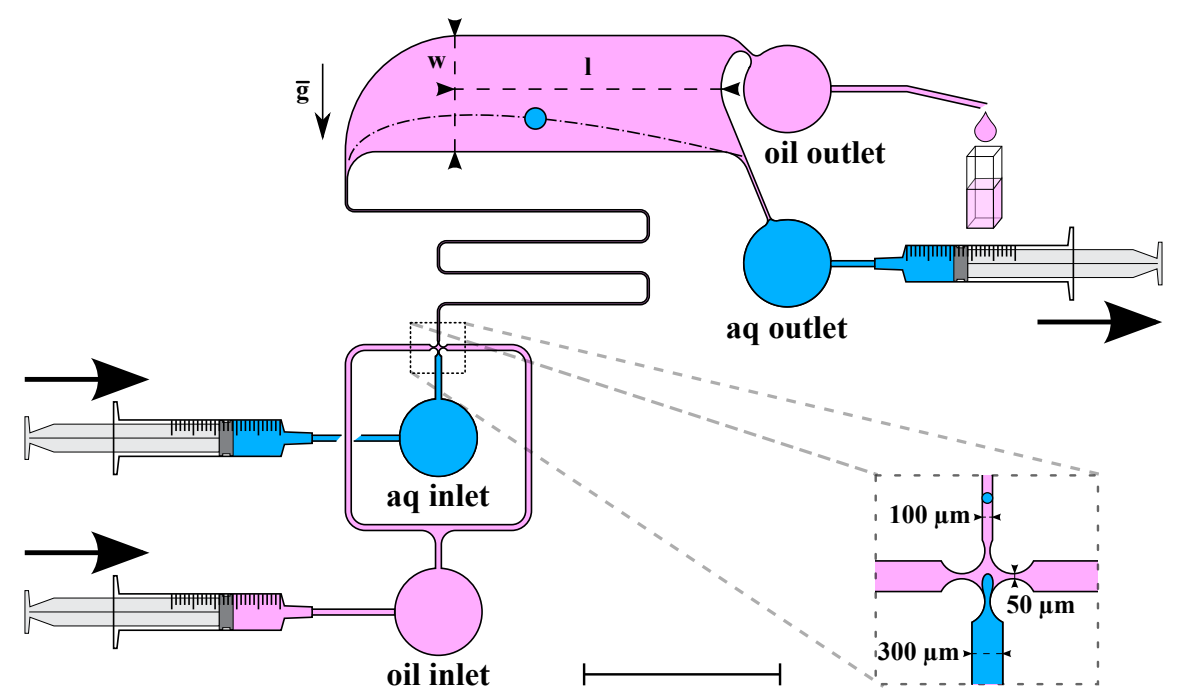

Figure 1: Scale drawing of the microfluidic platform used for droplet-based liquid-liquid extraction (the syringes and the cuvette at the oil outlet are not to scale). Droplets are formed at a flow focusing geometry and then inserted into a large separation chamber where gravity $(\bar{g})$ enables separation of the two phases. Blue denotes the aqueous (aq) phase and pink denotes the oil phase. The width, $w$, of the separation chamber is $6 \mathrm{~mm}$, its length, $l$, is $13.7 \mathrm{~mm}$ and the channel height is $100 \mu \mathrm{m}$ throughout. The width of the channels connecting to both the outlets is $150 \mu \mathrm{m}$. The oil phase was collected in a cuvette at the oil outlet and absorbance measurements were performed as detailed in Section 2.3. The scale bar in the main figure is $10 \mathrm{~mm}$ and the enlarged area shows the dimensions of the flow focusing geometry used for droplet generation. Single-headed arrows denote the directions of flow from the syringe pumps and of gravity. The images of syringes and the cuvette are from Wikimedia.org and are used under a creative commons license.

As described in detail in Section S1, if gravity is to enable an aqueous droplet suspended in octanol to migrate at least the entire width of the separation chamber relative to the continuous phase, the following inequality must be fulfilled:

$$
\frac{\tilde{d}^{2} \tilde{h} \tilde{l}}{\tilde{Q}_{t o t}} \geq 18 N_{\mathrm{St}}
$$

where $\tilde{d}$ is the dimensionless droplet diameter, $\tilde{h}$ is the dimensionless height of the sep- 
aration chamber, $\tilde{l}$ is the dimensionless chamber length, $\tilde{Q}_{\text {tot }}$ is the dimensionless total flow rate, $N_{\mathrm{St}}$ is the Stokes number (the ratio of viscous to gravitational forces). Therefore, in the microfluidic platform used herein, where $h=100 \mu \mathrm{m}, w=6 \mathrm{~mm}, l=13.7 \mathrm{~mm}$ and $d \leq h$, the total flow rate should be kept smaller than $20.8 \mu \mathrm{L} / \mathrm{min}$. It is important to note that this value defines the maximum flow rate, since smaller droplets will have a lower terminal velocity and will hence require a longer residence time in the separation chamber to allow for a channel width migration. Furthermore, the Bond number, $B o$, is $B o \leq 0.0031$, confirming that surface tension forces dominate in our platform and hence droplets are round (for details of the interfacial tension measurements used to determine the Bo see Section S3 in the SI).

\subsection{Chip fabrication}

Micromilling. A structured mould insert for injection moulding was designed in Autodesk Inventor Professional 2012, converted to G-code in CimatronE10 and fabricated in 2017 aluminium alloy (MetalCentret, Denmark) by micro milling (NTI CADcenter A/S, Denmark). Energy directors for ultrasonic welding ${ }^{16}$ were defined using a $60^{\circ}$ helical engraving tool (\#7025, DIXI polytool, Herstad+Piper, Denmark) producing 50 mm high prism-shaped energy directors.

Injection moulding. The aluminium mould insert and a matching counter-mould with 12 ISO Luer-fittings ${ }^{17}$ were installed in a Victory 80/45 Tech injection moulder (Engel, Austria). The polymer used for injection moulding was COC (grade 5013L-10, TOPAS Advanced Polymers, Germany) with a glass transition temperature $(T g)$ of $135{ }^{\circ} \mathrm{C}$. The injection temperature of the polymer was $270{ }^{\circ} \mathrm{C}$, and the mould and demoulding temperatures were kept stable at $120^{\circ} \mathrm{C}$.

Ultrasonic welding. To seal the microfluidic device, a $152 \mu \mathrm{m}$ thick COC sheet (grade 5013S-04, TOPAS Advanced Polymers, Germany) was bonded to the injection moulded piece using a Telsonic-USP4700 ultrasonic welder (Telsonic, Herstad+Piper, Denmark). The welding was conducted using $45 \mathrm{~J}$ at $90 \%$ amplitude and $20 \mathrm{kHz}$, with a trigger force on 
the piece of $600 \mathrm{~N}$ in the normal direction.

Annealing. To avoid solvent-induced cracking, internal stress from the isothermal injection moulding and ultrasonic welding was removed by annealing; The entire batch of chips (200 pieces) was loaded into an oven which was cycled from room temperature to $125^{\circ} \mathrm{C}$ and back to room temperature over a period of 2 hours. A comparison of solvent-induced stress cracking in annealed and non-annealed chips due to octanol exposure is shown in Section S5 of the SI.

Overall, including handling, the total turn-around time per chip was 40 seconds for injection moulding, 20 seconds for ultrasonic welding and 36 seconds for annealing, which amounts to a fabrication time of less than 2 minutes per device.

\subsection{Liquid-liquid extraction}

Standards for determining quinine extinction coefficients were prepared by dissolving quinine hydrochloride dihydrate ( $\geq 99.0 \%$, Sigma-Aldrich, Switzerland) either in 1x DPBS (Dulbecco's phosphate-buffered saline, pH 7.4, Life Technologies, Switzerland), or in octan-1-ol ( $\geq 99.0 \%$, Alfa Aesar, Germany) with $3 \%$ (w/w) ABIL EM90 (cetyl dimethicone copolyol, Rose Chemicals, UK). The DPBS and octanol used in the preparation of the standards were stored with $5 \%$ octanol and DPBS respectively to ensure mutual saturation of the two phases.

Samples for 'bulk' analyses were prepared in glass vials with aqueous to octanol (aqueous:octanol) volume ratios of $2: 1,1: 1,1: 2,1: 4,1: 8,1: 16,1: 32$ and 1:10, the latter of which creates a bulk sample with a volume ratio similar to the flow rate ratio in the droplet-based experiments. The total volume for each sample was $4 \mathrm{~mL}$ and all eight concentrations were prepared in triplicate, for a total of 24 samples. All aqueous and octanol volumes were calculated from mass measurements and density to ensure consistency. At the start of each experiment, the aqueous solution contained $1 \mathrm{mM}$ quinine hydrochloride dihydrate.

Droplet-based liquid-liquid extraction was conducted on the microfluidic platform de- 
scribed above using three Aladdin syringe pumps (AL-1000, WPI-Europe, UK) mounted with $500 \mu \mathrm{L}$ Hamilton Gas-tight syringes (VWR, Denmark), two for controlling the inlet flow rates and one for controlling the aqueous outlet flow rate. Assuming the system is operated under stable flow for 30 to 90 minutes per experiment, the oil outlet flow rate was assumed to satisfy the continuity equation $0=Q_{a q, \text { in }}+Q_{\text {oil, in }}+Q_{a q, \text { out }}+Q_{\text {oil,out }}$, where subscripts $a q$ and oil denote aqueous and oil phases respectively, and subscripts in and out denote the direction of flow as 'into' and 'out of' the microfluidic device respectively. The aqueous outlet flow rate, $Q_{a q, o u t}$, was set to $3.5 \mu \mathrm{L} / \mathrm{min}$ and this ensures that no droplets exit from the oil outlet, that the flow at the oil outlet is stable and that enough oil can be collected to finish the experiment within 30 minutes. During experiment, the microfluidic device was mounted such that the width of the separation chamber was parallel to the gravitational force (see $\vec{g}$ in Figure 1). The absorbance of the oil phase was measured by collecting a set volume of oil from the oil outlet and performing off-line measurements at 340 nm on a BioPhotometer plus (Eppendorf, Switzerland) using UVette cuvettes (Eppendorf, Switzerland). For the measurement of absorbance values in the range of 0.05 to 1.00 , both the $10 \mathrm{~mm}$ and the $2 \mathrm{~mm}$ path lengths of the cuvettes were used. Quinine concentrations determined from absorbance measurements using the Beer-Lambert law were used to calculate $D$ (see SI for further information). The parameters used for on-chip liquid-liquid extraction are summarised in Table 1.

Table 1: Parameters for on-chip liquid-liquid extraction. Symbols $(+)$ and $(\mathbf{x})$ denote datasets of unique $r_{Q}$ and will be used for identification in Figures 3 and 4.

\begin{tabular}{lcc} 
& $(+)$ & $(\mathbf{x})$ \\
\hline$Q_{a q, i n}(\mu \mathrm{L} / \min )$ & 0.5 & 1.0 \\
$Q_{\text {oil }, \text { in }}(\mu \mathrm{L} / \mathrm{min})$ & 5.0 & 5.0 \\
$Q_{a q, \text { out }}(\mu \mathrm{L} / \mathrm{min})$ & 3.5 & 3.5 \\
$r_{Q}=Q_{\text {oil }, \text { in }} / Q_{a q, i n}$ & 10 & 5 \\
Quinine concentration $(\mu \mathrm{M})$ & $100,250,500,750,1000$ \\
Number of experiments & 3 & 3 \\
Total number of devices & 15 & 15
\end{tabular}

On-chip droplet imaging was performed using a Manta G046B ASG camera (Allied Vi- 
sion, Denmark) mounted on a $1-4 \mathrm{x}$ adjustable zoom lens. Images were subsequently analysed using a custom made MATLAB algorithm incorporating circle detection by Circular Hough Transform to determine droplet size.

\section{Results and Discussion}

\subsection{Characterisation of the microfluidic platform}

The performance of the microfluidic platform over a range of aqueous and oil flow rates is illustrated in Figure 2 in terms of droplet size statistics. During initial device characterisation, it was found that configurations of $Q_{a q, i n} / Q_{o i l, i n}>0.2$ produced too many droplets for efficient droplet migration and hence separation of the phases at the device outlets. Since droplets at these flow rates do not leave the chamber quickly enough, the high droplet density effectively constricts the chamber dimensions, causing new droplets to have higher flow rates and thus lower residence times. This phenomenon is related to hydraulic damming and practically means that droplets agglomerate at the aqueous outlet causing new droplets to exit via the oil outlet. The box in Figure 2 highlights the maximum flow rate $\left(Q_{t o t}=Q_{a q, i n}\right.$ $\left.+Q_{o i l, i n}=20 \mu \mathrm{L} / \mathrm{min}\right)$ and inlet flow rate ratio $\left(Q_{a q, i n} / Q_{o i l, i n}=0.2\right)$ at which droplets can be separated from the oil flow at the maximum droplet diameter $(d=h)$. This is the region in which $\tilde{s}_{w} \leq \tilde{w}$ and the boundaries of the box depends on the droplet size because smaller droplets have a lower terminal velocity (further details can be found in Section S1 of the SI). Figure 2 clearly shows the robustness of the microfluidic platform in terms of the variety of flow rate combinations at which stable droplet production is possible.

The inter-device droplet production reproducibility was measured in triplicate under the experimental conditions described in Table 1. The statistical analysis of these data is summarised in Table 2. It is evident that the produced droplets have a high polydispersity $\left(\overline{C V_{D}} \sim 34 \pm 8.1 \%\right)$ but that average droplet diameters are conserved across different microfluidic devices $\left(S E M_{\bar{d}} \sim 15 \mu \mathrm{m}\right)$. As shown in Figure 1, the flow-focusing geometry 


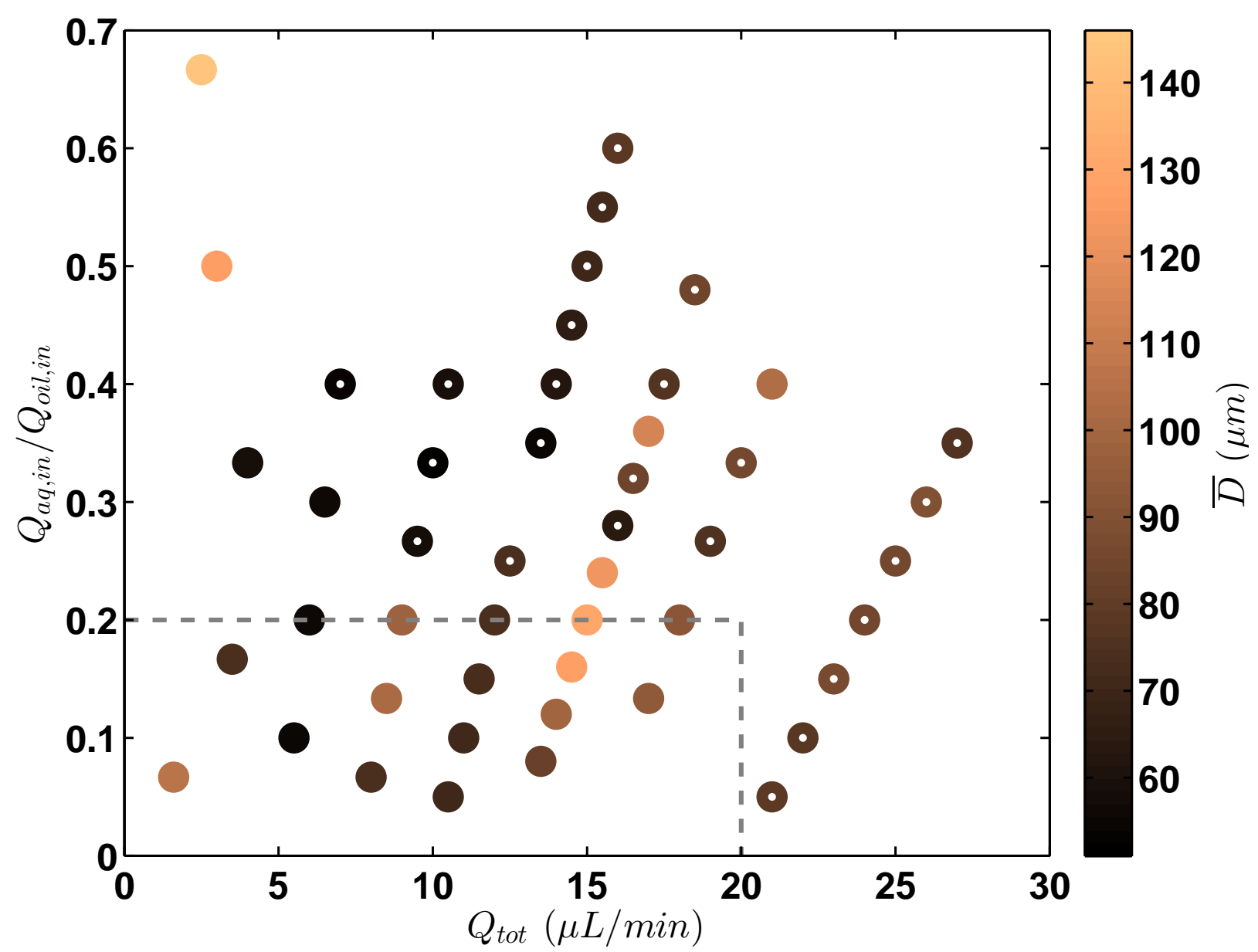

Figure 2: Characterisation of droplet formation on the microfluidic device. Average droplet diameter (colour map) versus total flow rate, $Q_{t o t}$, and versus the flow rate ratio, $Q_{a q, i n} /$ $Q_{o i l, i n}$. Data points with a white dot represent system conditions where the droplet residence time is too low to allow phase separation, calculated using Equation S2. The section highlighted by the dashed rectangle denotes the parameter space where flow rate combinations allow efficient droplet guidance. Note that the separation chamber has a height of $100 \mu \mathrm{m}$ and hence droplets with a larger diameter are non-spherical. 
used for droplet creation has rounded edges, which explains the high polydispersity when compared to other microfluidic platforms where the droplet formation geometry has sharp corners. These rounded edges are caused by a limitation of the milling process which, unlike lithographic methods, defines geometries by material removal using milling tools of finite diameters $(200 \mu \mathrm{m})$. Additionally, micromilling introduces surface roughness, which may also introduce variations during droplet break-off and formation.

Table 2: Droplet size statistics for on-chip liquid-liquid extraction experiments, where $\overline{C V_{D}}$ is the average coefficient of variation of droplet diameters, $\overline{\bar{d}}$ represents the grand mean (the arithmetic mean of the arithmetic mean) of droplet diameters and $S E M_{\bar{d}}$ is the standard error of the arithmetic mean of droplet diameters. The statistical analysis is based on droplet images acquired in the experiments described in Table 1 and each image contained a minimum of 50 droplets (on average there were 384 droplets per image). Each set of flow rate conditions was analysed in 15 individual experiments on 15 different microfluidic platforms.

\begin{tabular}{ccccc}
$Q_{a q, i n}(\mu \mathrm{L} / \mathrm{min})$ & $Q_{o i l, i n}(\mu \mathrm{L} / \mathrm{min})$ & $\overline{C V_{D}}(\%)$ & $\overline{\bar{d}}(\mu \mathrm{m})$ & $S E M_{\bar{d}}(\mu \mathrm{m})$ \\
\hline 1.0 & 5.0 & $34.1 \pm 9.3$ & 70.9 & 19.7 \\
0.5 & 5.0 & $33.7 \pm 6.8$ & 66.2 & 9.55
\end{tabular}

\subsection{Liquid-liquid extraction}

To verify that on-chip liquid-liquid extraction was not limited by diffusion at low droplet residence times, extractions were conducted with aqueous quinine concentrations of $100 \mu \mathrm{M}$, $250 \mu \mathrm{M}, 500 \mu \mathrm{M}, 750 \mu \mathrm{M}$ and $1 \mathrm{mM}$, at aqueous flow rates of $1 \mu \mathrm{L} / \mathrm{min}$ and $0.5 \mu \mathrm{L} / \mathrm{min}$ and with a constant oil flow rate of $5 \mu \mathrm{L} / \mathrm{min}$ throughout. The distribution of quinine between the aqueous and oil phases is described by the relationship between the inbound aqueous mass flow rate $\left(I_{a q, i n}\right)$ and the outbound oil mass flow rate $\left(I_{\text {oil }, \text { out }}\right)$, as shown in Figure 3 , where $I=c Q$ and $c$ is concentration. Since the outbound mass flow rate is observed to scale linearly with the inbound mass flow rate, the system is at equilibrium when the phases are separated for analysis. If the residence time of the droplets in the separation chamber was insufficient to allow for mass transfer equilibrium, variations in the inbound mass flow rate introduced by varying the flow rate (and hence the residence time of the droplets) or the aqueous quinine concentration, would result in a non-linear relationship between $I_{\text {oil,out }}$ 
and $I_{a q, i n}$. The two total flow rates used in this study of 5.5 and $6.0 \mu \mathrm{L} / \mathrm{min}$ cause average residence times of 129 and 118 seconds respectively. The fastest droplets shown in Video S1 have a residence time of 68 seconds. Our average residence times are at least one order of magnitude more than what was needed to achieve steady-state in diffusion studies of fluorophores in larger droplets (equilibrium was reached in less than 8 seconds). ${ }^{7,18}$

In order to assess the performance of liquid-liquid extraction on the droplet microfluidic platform compared to standard methodologies, the distribution coefficients of quinine from bulk overnight experiments and on-chip liquid-liquid extractions are compared in Figure 4. For bulk experiments, this figure shows the distribution coefficients determined at the volume ratios stated in the Materials and Methods section. Equation 2 was used to calculate the apparent acid dissociation constant, $p K^{\prime},{ }^{19}$ which is unique to the system ( $\mathrm{pH}$ and buffer) and, unlike the distribution coefficient, is independent of the volume ratio.

$$
D=\frac{V_{a q}}{V_{o i l}}\left(10^{p K_{a}-p K^{\prime}-1}\right)
$$

Here $V$ is volume and $p K_{a}$ is the true acid dissociation constant. Table 3 summarises experimental values of $p K^{\prime}$ both from bulk and on-chip droplet based liquid-liquid extraction experiments, and from literature. The $p K_{a}$ value used for calculating $p K^{\prime}$ from Equation 2 was 8.505 , which defines the average of two literature values, $8.58^{20}$ and $8.43 .^{21}$

Table 3: Comparison of experimental data gathered from bulk and microfluidic experiments with literature values. The apparent acid dissociation constant, $p K^{\prime}$, was calculated from volume or flow rate ratios, $r_{V}$ or $r_{Q}$, using $D$ at $\mathrm{pH} 7.4$, a $p K_{a}$ value of $8.505^{20,21}$ and Equation 2. Markers $(O),(+),(\mathbf{x})$ and $(\mathrm{o})$ used in the table refer to Figure 4. Error estimations in the $p K^{\prime}$ values refer to the standard deviation. Values for $D$ as measured from bulk and microfluidic experiments in this table refer to the data in Figure 4 because $D$ is dependent on the volume ratios (unlike $p K^{\prime}$, which is hence a better standard for inter-system comparison).

\begin{tabular}{lccc} 
& $r_{V}$ or $r_{Q}$ & $D$ & $p K^{\prime}$ \\
\hline Data from bulk experiments, $(\odot)$ & $1 / 2$ to $32 / 1$ & Fig. 4 & $7.25 \pm 0.58$ \\
Data from on-chip experiments, $(+)$ and $(\mathbf{x})$ & $5.0 / 1.0$ and 5.0/0.5 & Fig. 4 & $7.24 \pm 0.15$ \\
Data from literature, ${ }^{20}(\circ)$ & 1 & 1.97 & 7.21
\end{tabular}

As is evident from the data presented in Table 3, there is a good agreement between the 


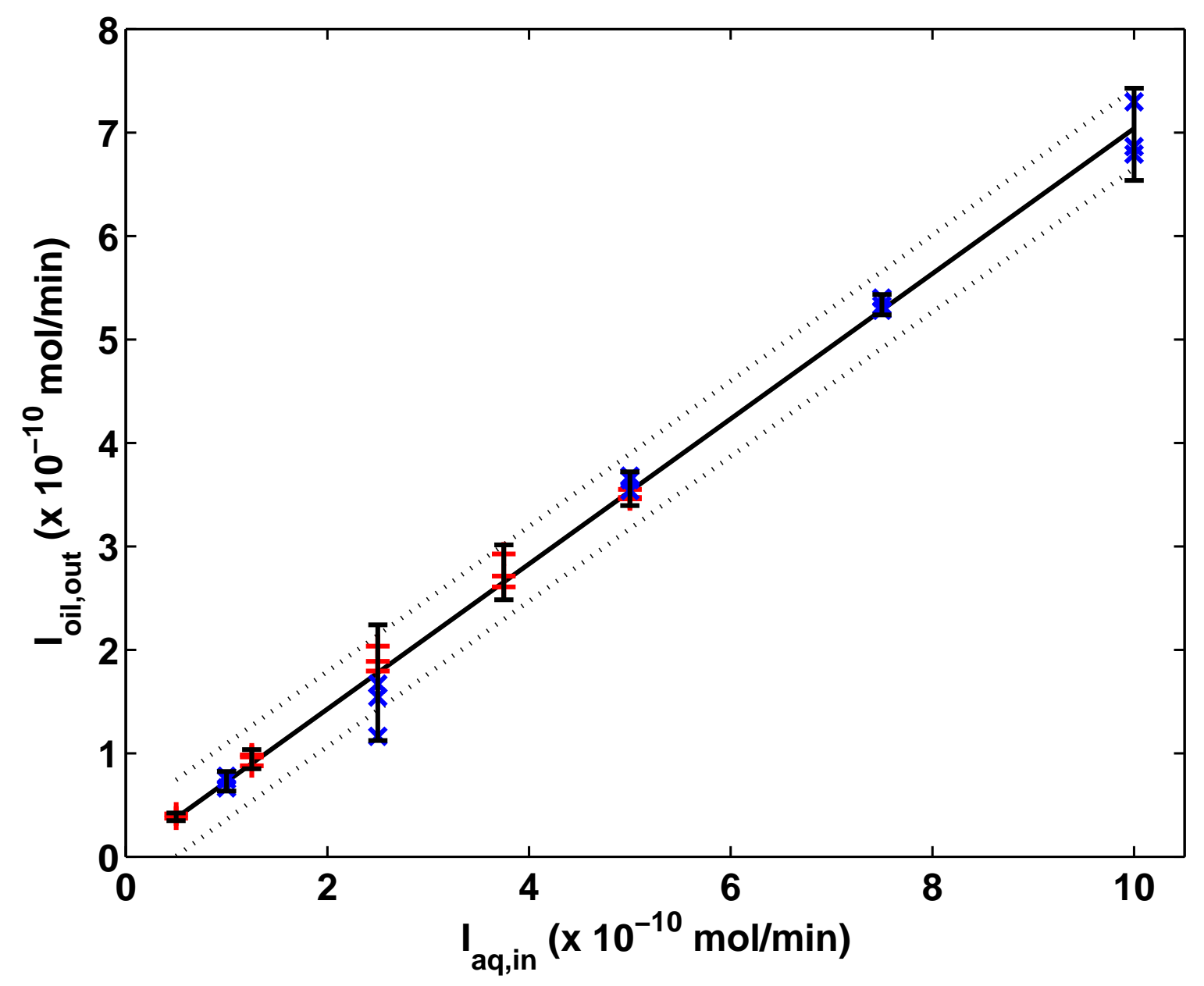

Figure 3: Outbound versus inbound molar flow rates for on-chip liquid-liquid extraction. The inbound molar flow rate is varied by adjusting the aqueous flow rate and hence the aqueous quinine concentration. $(+)$ and $(\mathbf{x})$ represent aqueous flow rates of $0.5 \mu \mathrm{L} / \mathrm{min}$ and $1.0 \mu \mathrm{L} / \mathrm{min}$ respectively. The concentration of quinine was calculated using extinction coefficient data shown in Figure S3. Error bars show 2 standard deviations from the mean. Solid (-) and dotted (..) lines represent the linear fit and prediction intervals of the data respectively. Prediction intervals were calculated using the MATLAB package polyconf and illustrate the upper and lower bounds in which $95 \%$ of future data points will fall. 


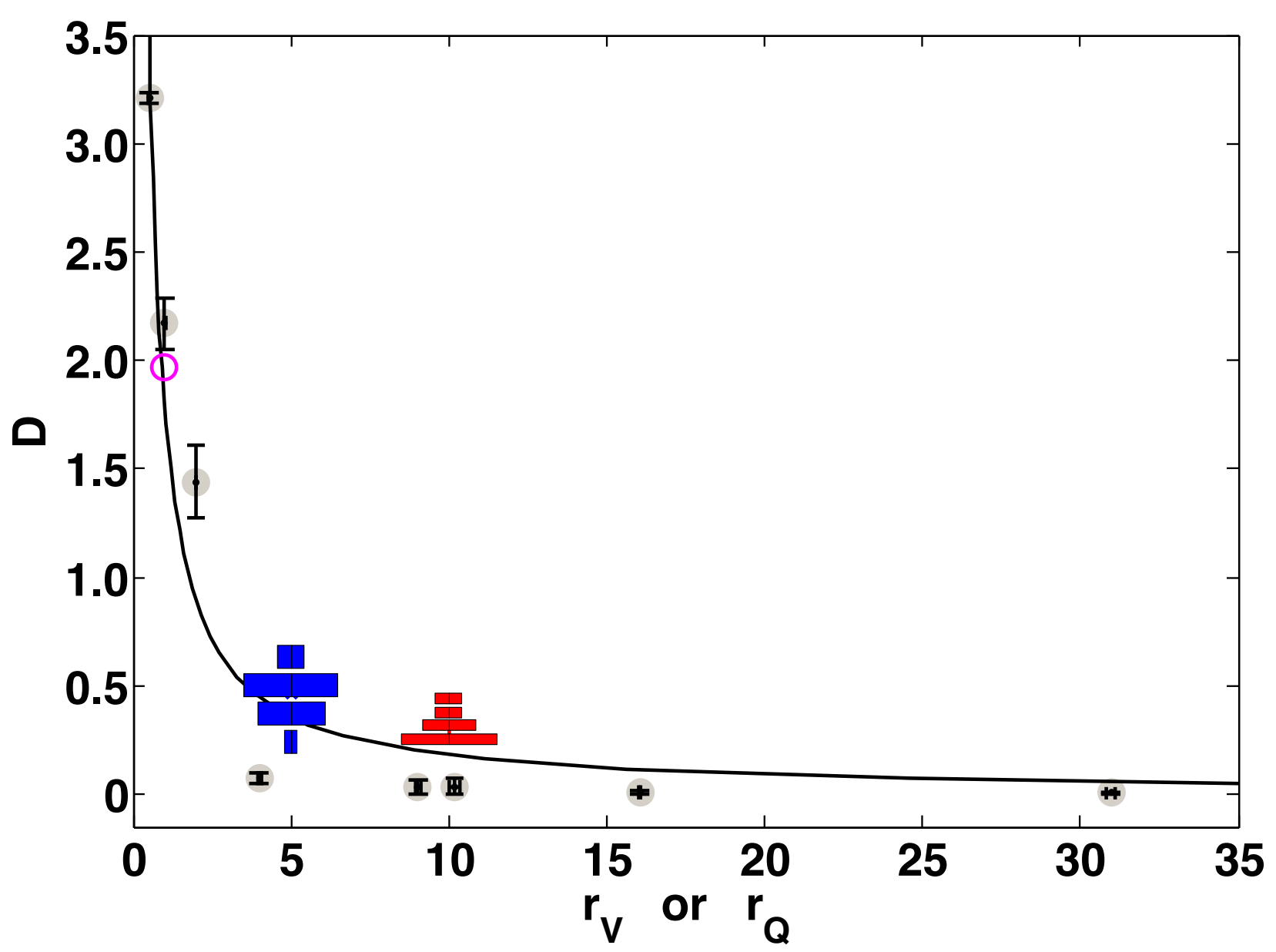

Figure 4: Distribution coefficients, $D$, both from bulk experiments ( $)$ plotted versus volume ratio, $r_{V}=V_{\text {oil }} / V_{a q}$, and from on-chip liquid-liquid extraction experiments at aqueous flow rates of $0.5 \mu \mathrm{L} / \mathrm{min}$ (red violin plot) and $1.0 \mu \mathrm{L} / \mathrm{min}$ (blue violin plot) plotted versus the volume flow rate ratio, $r_{Q}=Q_{\text {oil,in }} / Q_{a q, i n}$. The concentration of quinine was calculated using extinction coefficient data shown in Figure S3. The distribution of data points from on-chip experiments are illustrated as violin plots to highlight the large amount of measurements gathered for each data point when using a microfluidic platform (each violin plot incorporates data gathered from 15 separate experiments as detailed in Table 1). The solid line represents the least squares fit of Equation 2 for the data from bulk experiments, $D=\left(10^{p K_{a}-p K^{\prime}-1}\right)$ $1 / r_{V}=10^{0.25} 1 / r_{V}$. (o) represents literature data. ${ }^{20}$ 
$p K^{\prime}$ values measured in bulk and on-chip experiments, and these values are also in good agreement with literature. Table 4 shows how other parameters such as cost and reagent consumption compare between microfluidic and traditional methodologies. In terms of cost efficiency (reagent consumption and time) and sensitivity to user interference, the microfluidic platform greatly outperforms traditional methodologies. Furthermore, it is possible to perform continuous measurements using the microfluidic platform, such as in-line absorbance measurements and, during one continuous experiment in the microfluidic platform the variation of combinations of $Q_{a q, i n}$ and $Q_{o i l, i n}$ allows for the concurrent acquisition of multi-parameter data $\left(e . g . r_{Q}, D\right)$.

Table 4: Comparison of parameters associated with bulk and on-chip methodologies for liquid-liquid extraction. N/A denotes not applicable.

\begin{tabular}{lcc} 
& Bulk & On-chip \\
\hline Experiment time & $>24 \mathrm{~h}$ & $30 \mathrm{~min}$ \\
Reagent volume & $>20 \mathrm{~mL}$ & $300 \mu \mathrm{L}$ \\
Cost of reagents & $25 \mathrm{USD}$ & $0.37 \mathrm{USD}$ \\
Cost of microfluidic device & $\mathrm{N} / \mathrm{A}$ & $1 \mathrm{USD}$ \\
Sensitivity to user interference & High & Low \\
Repeatability & Good & Good \\
Continuous measurements & Not possible & Possible \\
Phase space sweep & Not possible & Possible
\end{tabular}

\section{Conclusions}

The microfluidic devices presented herein are fabricated using injection moulding, ultrasonic welding and annealing, which are all processes currently used in commercial manufacture, with a time per device of less than 2 minutes. We demonstrate the reliability of the devices by performing experiments on 30 different devices, with distribution coefficient data gathered on these platforms showing close agreement with literature values and low statistical variation under a variety of flow conditions and concentrations. To enable the use of this platform in non-specialist laboratory settings, the device design is simple and gravity is used to enable 
phase separation. Finally, we assess the parameters associated with this microfluidic platform as compared to the shake-flask method. We show that our microfluidic platform is 48 times faster and uses $99 \%$ less reagents than the shake-flask method. In addition, user sensitivity and contamination, which are sources of error for conventional techniques, are negligible in our platform. As future work, the system will be integrated with on-line absorption detection to allow extraction of data from individual droplets to enhance analytical efficiency.

\section{Acknowledgements}

This work was partially funded by The Danish Council for Independent Research (Grant number 09066477). Carl Esben Poulsen would like to thank The Oticon Foundation, The Otto Mønsted Foundation and The Augustinus Foundation for funding.

\section{Supporting Information Available}

Additional supporting information is available online: a mathematical description and a two-dimensional numerical (COMSOL) model of the effect gravity has on droplets in the separation chamber, experimental data to verify our model, absorbance data at different quinine concentrations and equations used to calculate the extinction coefficient of quinine, interfacial tension measurements of our octanol/DPBS/Abil EM90 system required to calculate the Bond number, derivations of the equations used for calculating $D$ from quinine concentrations and data to show the effect that annealing has on ultrasonic welded devices. This information is available free of charge via the Internet at http://pubs.acs.org/.

\section{References}

(1) OECD Guidelines for the Testing of Chemicals: Partition Coefficient ( $n$ octanol/water): Shake Flask Method; Organisation for Economic Co-operation and Development, 1995. 
(2) Alimuddin, M.; Grant, D.; Bulloch, D.; Lee, N.; Peacock, M.; Dahl, R. J. Med. Chem. 2008, 51, 5140-5142.

(3) Elvira, K. S.; Casadevall i Solvas, X.; Wootton, R. C. R.; deMello, A. J. Nat. Chem. 2013, 5, 905-915.

(4) Soares, R.; Novo, P.; Azevedo, A.; Fernandes, P.; Aires-Barros, M. R.; Chu, V.; Conde, J. P. Lab Chip 2014, 14, 4284-4294.

(5) Xiao, H.; Liang, D.; Liu, G.; Guo, M.; Xing, W.; Cheng, J. Lab Chip 2006, 6, 10671072.

(6) Marine, N. A.; Klein, S. A.; Posner, J. D. Anal. Chem. 2009, 81, 1471-1476.

(7) Mary, P.; Studer, V.; Tabeling, P. Anal. Chem. 2008, 80, 2680-2687.

(8) Miyaguchi, H.; Tokeshi, M.; Kikutani, Y.; Hibara, A.; Inoue, H.; Kitamori, T. J. Chromatogr. A 2006, 1129, 105-110.

(9) Kumemura, M.; Korenaga, T. Anal. Chim. Acta 2006, 558, 75-79.

(10) Chen, H.; Fang, Q.; Yin, X.-F.; Fang, Z.-L. Lab Chip 2005, 5, 719-725.

(11) Kralj, J. G.; Sahoo, H. R.; Jensen, K. F. Lab Chip 2007, 7, 256-263.

(12) Kralj, J. G.; Schmidt, M. A.; Jensen, K. F. Lab Chip 2005, 5, 531-535.

(13) Goyal, S.; Desai, A. V.; Lewis, R. W.; Ranganathan, D. R.; Li, H.; Zeng, D.; Reichert, D. E.; Kenis, P. J. Sens. Actuators, B 2014, 190, 634-644.

(14) Wagli, P.; Chang, Y.; Homsy, A.; Hvozdara, L.; Herzig, H. P.; de Rooij, N. F. Anal. Chem. 2013, 85, 7558-7565.

(15) Baret, J.-C. Lab Chip 2012, 12, 422-433. 
(16) Kistrup, K.; Poulsen, C. E.; Østergaard, P. F.; Haugshøj, K. B.; Taboryski, R.; Wolff, A.; Hansen, M. F. J. Micromech. Microeng. 2014, 24, 125007.

(17) Andresen, K. Ø.; Hansen, M.; Matschuk, M.; Jepsen, S. T.; Sørensen, H. S.; Utko, P.; Selmeczi, D.; Hansen, T. S.; Larsen, N. B.; Rozlosnik, N.; Taboryski, R. J. Micromech. Microeng. 2010, 20, 055010.

(18) Yu, J.; Chin, L.; Chen, Y.; Zhang, G.; Lo, G. MicroTAS 2010 2010, 1079-1081.

(19) Clarke, F. H. J. Pharm. Sci. 1984, 73, 226-230.

(20) Warhurst, D. C.; Craig, J. C.; Adagu, I. S.; Meyer, D. J.; Lee, S. Y. Malar. J. 2003, 26.

(21) Schulman, S. G.; Threatte, R. M.; Capomacchia, A. C.; Paul, W. L. J. Pharm. Sci. 1974, 63, 876-880. 


\section{Graphical TOC Entry}

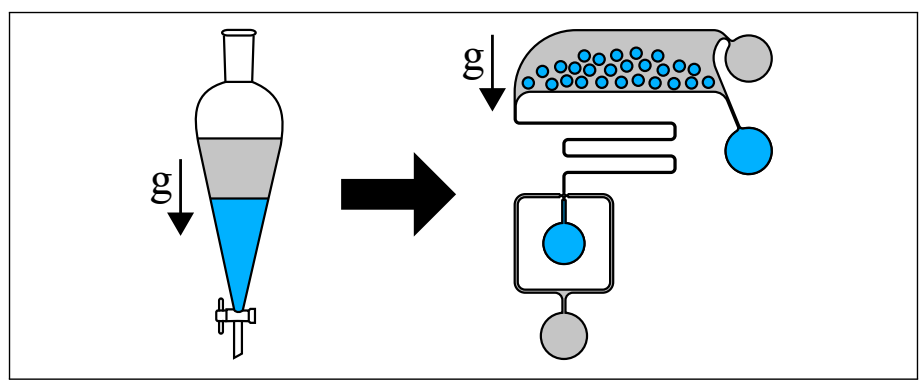

14

15

16

17

18

19

20

21

22

23

24

25

26

27

28

29

30

31

32

33

34

35

36

37

38

39

40

41

42

43

44

45

46

47

48

49

50

51

52

53

54

55

56

57

58

59

60 